\title{
Smart Street Light Monitoring using Iot
}

\author{
Sangameshwar, Akhila Sagi, Hareeshwar Channapragada, N Preethi, V V Mounika
}

\begin{abstract}
This paper is aimed at defining the process of automating the operation of electronic devices, in this case, street lights of a certain campus or area where there is a reliable network connection. This is achieved by using a microcontroller to control the target electronic device wirelessly instead of operating the electronic device manually and a relay to electromagnetically provide the functioning of a switch and also use a step-down converter to regulate voltage and convert it from 220v AC to 5v DC. The microcontroller used is the ESP8266 (NodeMCU) as this microcontroller has inbuilt Wi-Fi module that can suffice connecting to a network helping us in reaching the goal of wirelessly operating electronic devices. This is a step in the direction of a smart world where internet of things is the way of communication between all electronic devices and efficient utilization of this system saves a lot of energy and there will be lesser need of human intervention. This is an efficient step in the right direction of digitizing routine tasks and have much more control over them as opposed to being present physically near the electronic device.
\end{abstract}

Keywords: Internet of Things, Blynk IoT service, NodeMCU, Streetlight monitoring, Regulator.

\section{INTRODUCTION}

Internet of Things is an emerging technology which allows for connection of various electronic devices such that otherwise would have been controlled manually. Information sharing between these connected devices can be termed as a major advantage of using this technology [1][6]. In our daily routine, we observed that there are certain actions we perform in a sequence without giving much thought to it. We do not particularly look at a time to turn on our lights at our home, we just do it based on our perception of when the sun sets. This is a routine task and can therefore be converted into logic. This is the very foundation of our research. We wanted to question if it was possible to convert this set of actions performed in a sequence based on some conditions into a logic which we can feed into an automatic system for it to do our routine tasks such as operating street lights.This has two major advantages: firstly it is easier to operate the connected devices compared to manually operating them, and, secondly, in the present world of every

Revised Manuscript Received on April 25, 2020.

*Correspondence author

Mr. Sangameshwar*, Dept. of CSE, GITAM School of Technology, Bengaluru; Email: sbiradar@gitam.edu

Akhila Sagi, Dept. of CSE., GITAM School of Technology, Bengaluru; Email: akhila.sagi31@gmail.com

Hareeshwar C, Dept. of CSE, GITAM School of Technology, Bengaluru; Email: hareeshwar.c@gmail.com

N Preethi, Dept. of CSE, GITAM School of Technology, Bengaluru; Email: preethiyadav99@gmail.com

V V Mounika, Dept. of CSE, GITAM School of Technology, Bengaluru; Email: velavarthivmounika@gmail.com

(c) The Authors. Published by Blue Eyes Intelligence Engineering and Sciences Publication (BEIESP). This is an open access article under the CC BY-NC-ND license (http://creativecommons.org/licenses/by-nc-nd/4.0/) electronic device being connected to each other, it is only going to be more and more important to have electronic equipment connected to a digital medium to share information.

\section{LITERATURE SURVEY}

The existing system consists of an electrical wiring circuit that connects street lights with a power source and switches to operate the system [2]. The switch determines if the circuit is opened or closed and this switch, hence, acts as the control for the whole system. This system has to be manually operated from where the physical location of the system exists. This system cannot be controlled from anywhere other than the physical location of the switches provided for the system or the power source. It is also noticed that this system requires each of the switches for each of the corresponding streetlights to be operated individually. The potential of making the system smart is seen as the entire electronic circuit consists of manual switches which are used to operate the system. They can be considered as the control of the entire system [3]. Here, the possibility of remotely operating the switch and hence, control the entire system lead to the inception of this project.

\section{PROPOSED SYSTEM}

The proposed system involves connecting an extra switch mechanism to the already existing switch but the new switch should be able to connect to the internet to receive commands from a user [4]. This is possible by connecting the new switch in series with the already exiting switch and having the old switch always turned on. The new switch is the microcontroller we equip for the project i.e., NodeMCU. Since this microcontroller can be controlled wirelessly with a smartphone, the entire system becomes smart and it is no longer required to be manually operated from the physical location of the switch.

\section{A. Working principle of IoT}

Internet of Things is a godsent in terms of opening new possibilities of interacting with electronic devices by digitally interfacing them possibly providing information in a very simple user-friendly format to a smart device connected to the same network as the rest of the system. In this system, every device required to be operated on the basis of IoT, are connected to each other on the same network. This network can be any form of network like LAN, WAN, MAN or local Wi-Fi or even cables, although it is not advisable to have a large network of devices with cables as the medium of transport of the network as it causes so much mess of wires and defeats the simplicity achieved by opting for Internet Of things in the first place.

Published By:

Blue Eyes Intelligence Engineering

\& Sciences Publication

(C) Copyright: All rights reserved.

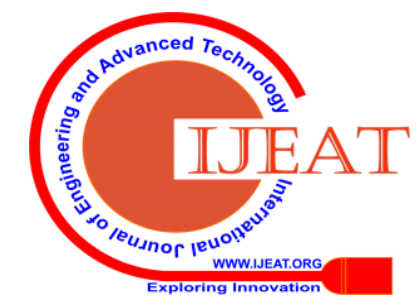




\section{Smart Street Light Monitoring using Iot}

This system is then interfaced by an end-user. The user can decide what kind of tasks must be performed by the system and the goal is to enable the user to have the privilege of giving these commands from anywhere in the world. It is a possibility since the entire world is connected with through the internet and this can be used as a medium to communicate information from the user to the devices that must be interacted with to send and receive data to perform some tasks required by the user. In this world of possibilities, anything can be connected to the internet with the help of supporting devices connecting to the network and acting as switches for the said mechanism to work. This enables, for say, to be able to turn on air conditioning in our car or our home knowing we will be there in a few minutes time to keep the atmosphere of the environment cool just as we enter. This can be further automated by connecting the

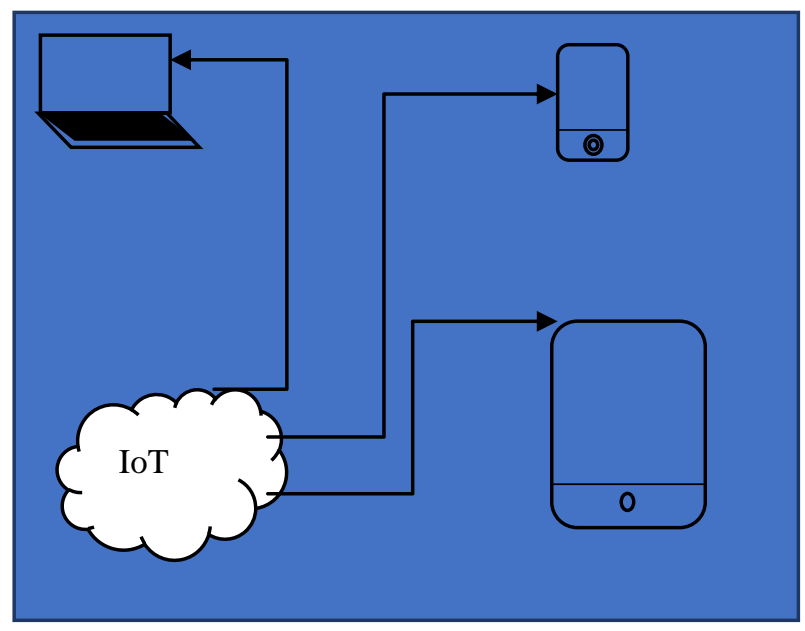

Figure 1. Internet of things

GPS available on our phones to send a message to the home air conditioner to turn on as and when there are say 10 mins left for the journey to complete and to reach home. This is just one of many possible ways of interconnecting various devices and software services to make one's life a lot easier and simpler. Any switch has only two states: it is either turned on or it is turned off. This can act as the very basic quality of digital devices in general. This can be used to our benefit as sending a binary signal is not a tough job for networks. The goal of Internet of Things is to one day have a seamless transition between all devices that can communicate with each other and make decisions for the user based on the user's regular habits and interests.

\section{B. Microcontroller}

Unlike humans, electronic devices do not have a brain of their own and so we need to instruct the devices what they should to do in order to cumulatively finish a larger task that all these smaller tasks contribute to. This is where a microcontroller comes into play. A microcontroller is a device that is essentially used to send digital or analogue signals as per requirements to electronic devices to have these electronic devices perform certain tasks as per needs and requirements. A microcontroller acts as the central processing unit of any electronic circuitry. In essence, a microcontroller is a scaled down version of a standard size CPU used in standalone personal computers, and it is used to control projects that require lesser power. Hence the name "micro" controller. In this project, we use the ESP8266 microcontroller, commonly known as "NodeMCU". The main reason NodeMCU is preferred over other microcontrollers is, for this project, we need connection to the cloud through a wireless network medium. The best way to execute this connection is to have the microcontroller connected to a wireless LAN network (Wi-Fi). NodeMCU has built-in network adapter that supports Wi-Fi connectivity. This is the main reason NodeMCU is most suitable for this project. We can program the microcontroller to connect to a specific SSID by providing the necessary details like SSID and password associated with that network. This makes our goal of being able to wirelessly operate electronic devices much simpler and easier.

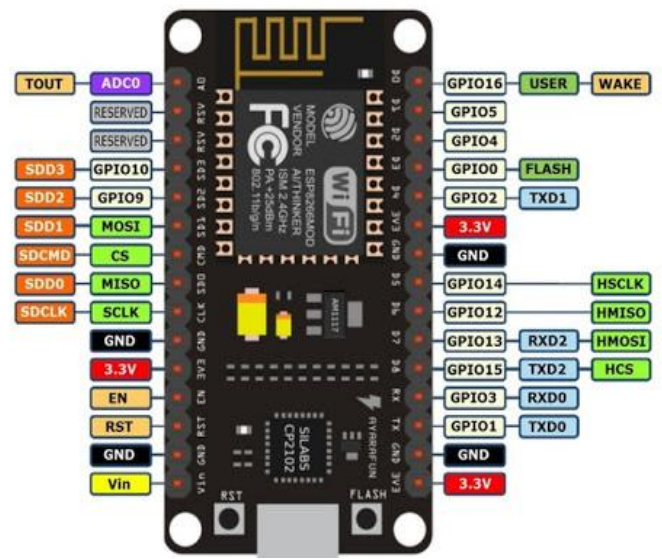

Figure 2. NodeMCU: Microcontroller

\section{Blynk IoT service}

In order to communicate wirelessly through distances longer than 100-150 meters, we need a server that provides us network access to be able to communicate with the devices from longer distances. We use the Blynk services to provide our project with the server that is online almost all the time [5], so that we can have a reliable connection between the two ends of the network and the ultimate goal of simplicity in operating the electronic devices wirelessly is achieved. This service is further enhanced with the help of android or iOS application provided by Blynk to interface the electronic device targeted to be controlled with the help of a microcontroller. This further simplifies the process of wirelessly controlling electronic devices as the setup requires us to connect the microcontroller to a laptop once to program the necessary wireless LAN details and any preset programming required to logically make the microcontroller work according to our needs, but once this setup is done, there will be no further need of a laptop until any updates are required to be made to the code. The microcontroller can be connected to the circuit and be controlled completely through the Blynk application.

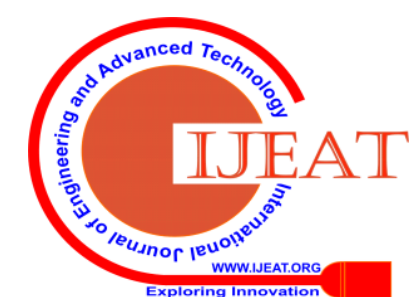




\section{FLOWCHART OF WORKING SYSTEM}

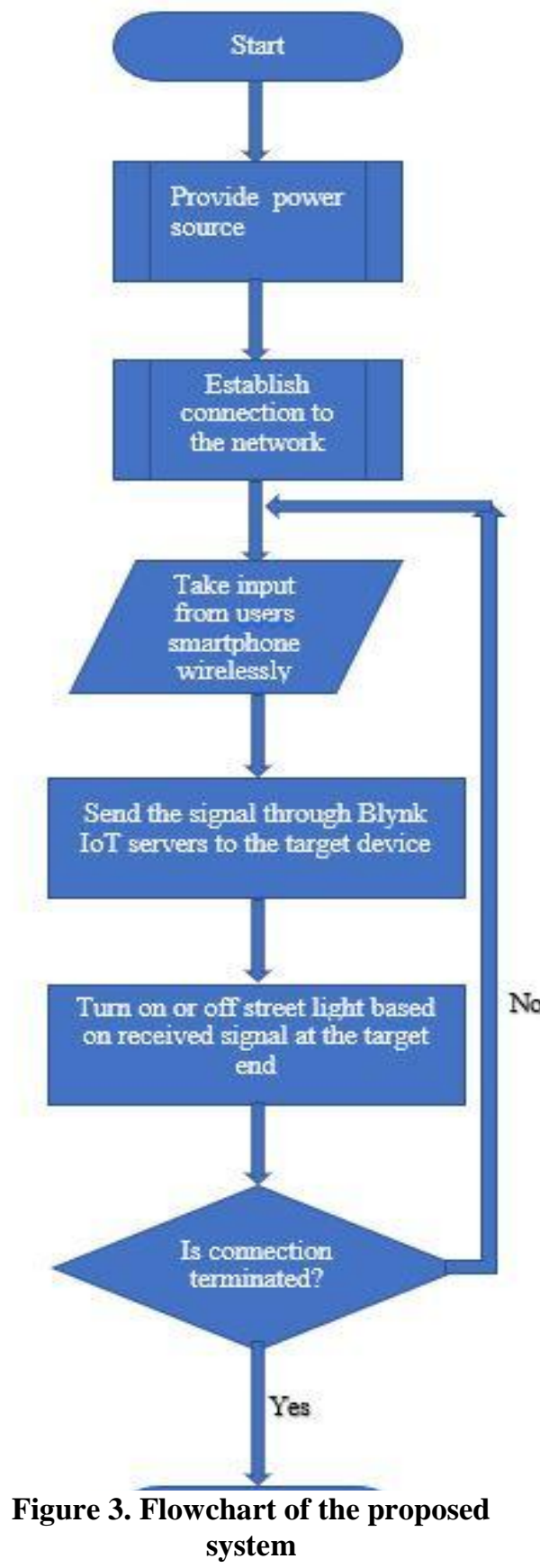

\section{RESULTS AND DISCUSSION}

The prototype displays the following results:

1. Connection is reliably established as soon as the system is powered.

2. The commands given by the user through phone are efficiently executed at the target end as intended.

3. The lights turn on or off based on command given by the user.

4. The system can be operated from anywhere in the world given that there is an active internet connection available.

5. The server is reliable and is online always.

The system can now be operated remotely from any location instead of being present in the location of the switch and operate it manually. It is a step in the direction of minimising time spent on daily routine tasks. To make it completely automated, we have to give it a logic to turn on or off based on sunset and sunrise times taken from a reliable weather forecast and that will make need of any human intervention minimal.

\section{CONCLUSION}

By implementing this system, we move one step closer to a smarter digital world by eliminating efforts in tasks that require us to manually operate them by being physically present near the target electronic equipment. This system is an efficient implementation of the internet of things concept as it makes it possible to wirelessly operate electronic devices like street lights from anywhere in the world where there is a network connection available. The system is easy to setup and implement and requires no extra maintenance compared to the already existing system. This system can be further enhanced by writing logic into the code that can be capable of retrieving information of the time of sunset and sunrise from a reliable weather reporting source and automate the process completely by turning on the street light at the time of sunset and turning it off by sunrise. This further eliminates human intervention and a manual visit to the location of the street lights will be required only in case of a malfunction.

\section{REFERENCES}

1. [Figure 1. Internet of Things] An image showing the pictorial representation of internet of things; Author: Hareeshwar C, date: $14 / 03 / 2020$

2. [Figure 2. NodeMCU: Microcontroller] This is an image of NodeMCU microcontroller available in google search results. https://www.google.co.in/url?sa=i\&url=https\%3A\%2F\%2Fwww.hicli part.com $\% 2$ Ffree-transparent-background-png-clipartbniig\&psig=AOvVaw3ankc6b6pJu0RRu3Iy4LWp\&ust $=1585392092$ 351000\&source=images\&cd=vfe\&ved=0CAIQjRxqFwoTCLiD6Pa7u ugCFQAAAAAdAAAAABAD

3. [Figure 3. Flowchart of the proposed system]A flowchart describing the entire process of the system; Author: Hareeshwar C, date: $14 / 03 / 220$

4. A. Zanella, N. Bui, A. Castellani, L. Vangelista and M. Zorzi, "Internet of Things for Smart Cities," in IEEE Internet of Things Journal, vol. 1, no. 1, pp. 22-32, Feb. 2014.

5. M. Durgun and L. Gökrem, "Cloud-Based Adjustable and Section Led Pattern Controlled Street Light," 2019 3rd Internationa Conference on Advanced Information and Communications Technologies (AICT), Lviv, Ukraine, 2019, pp. 308-310.

6. B. Kul, "IoT-GSM-based high-efficiency LED street light control system (IoT-SLCS)," 2017 XXVI International Scientific Conference Electronics (ET), Sozopol, 2017, pp. 1-5.

7. B. R. Prakash, S. Megha, M. K. Francis, S. Niharika and M. Megha, "Intelligent Management of Street Lights using Internet of Things and Power Management," 2019 International Conference on Smart Systems and Inventive Technology (ICSSIT), Tirunelveli, India, 2019, pp. 279-283.

8. S. Chen, G. Xiong, J. Xu, S. Han, F. Wang and K. Wang, "The Smart Street Lighting System Based on NB-IoT," 2018 Chinese Automation Congress (CAC), Xi'an, China, 2018, pp. 1196-1200.

9. U. Bhagat, N. Gujar and S. Patel, "Implementation of IOT in development of intelligent campus lighting system using mesh network," 2018 International Conference on Smart Systems and Inventive Technology (ICSSIT), Tirunelveli, India, 2018, pp. 251256.

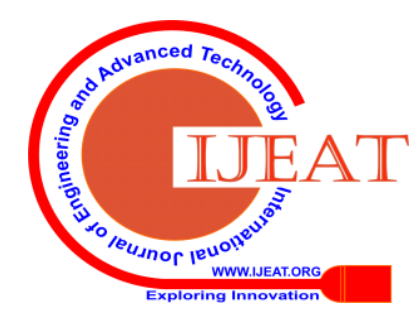




\section{Smart Street Light Monitoring using Iot}

\section{AUTHORS PROFILE}

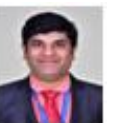

Mr. Sangameshwar, Sangameshwar obtained his Bachelors degree in Computer Science and Engineering from VTU Belagavi. Then he obtained his masters in Digital Communication and Networking from Dayananda Sagar College Engineering, Bengaluru. He is pursuing his $\mathrm{PhD}$ in IoT and Edge Computing from GITAM, Bengaluru. Currently, He is working as an Assistant Professor in Department of Computer Science \& Engineering, GITAM (Deemed to be University), Bengaluru. He has working experience of 9 years and 8 months.He has huge passion towards new technologies like IoT, AI/ML, Python, Data Science, Cyber security and lot more. but, his favourite technology remain IoT and Edge computing. He has done various certifications with respect to new technologies. He activitly participates in every technical events. He has won 2nd prize in cyber security curate-thon conducted by Nasscom. He has stood as a backbone for various projects.

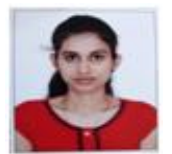

Akhila Sagi, Akhila Sagi is pursuing her Bachelor's degree in Computer Science and Engineering from GITAM (Deemed to be University), Bengaluru. Her areas of interest include Internet of Things, Machine learning, ethical hacking and logic building. She has participated in IoT workshops conducted in GITAM deemed to be university, participated in automation anywhere workshop, ethical hacking workshops and campus robotics workshop. She is a part of campus robotics club and she is a member in Computer Society of India. She has also participated in competitions held by hacker rank and hacker earth. She has worked on academic projects based on Internet of Things in college. She is certified by Automation Anywhere in RPA, certified by Techkriti, IIT Kanpur for participating in sixth sense technology workshop and certified in ethical hacking by Techkriti, IIT Kanpur.

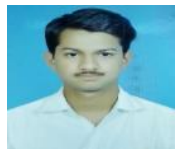

Hareeshwar Channapragada, Hareeshwar is currently pursuing his Bachelor's degree in Computer science and engineering from GITAM University, Benglauru. His areas of interest include logic building, design and analysis of algorithms and Machine learning. He has a gold badge (Rank 1) in C language in hackerrank. He has taken part in various coding competitions held by Google like the Google kick start and Google code Jam and several other competitions held by hackerrank. He has also taken part in workshop on Cyber security on the identification and testing of effects of various malwares held by K7 Security systems, participated in workshops on IoT held by GITAM University and is also a part of campus robotics club.

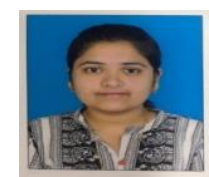

N Preethi, Preethi is pursuing her Bachelor's degree in Computer Science and Engineering from GITAM University, Bengaluru. She is currently working as an ISM in Intellipaat. She is proficient in finding and resolving the things and she also worked on academic project "Liquid level monitoring using IoT". She has participated in workshop on "Ethical Hacking and Cyber Security" at GITAM school of technology and she completed her internship on Web technology at Ridhan technologies Hyderabad, created her personal portfolio website using bootstrap, she developed a website for Himalaya Life Line (India) Limited. She has also participated in campus robotic club workshop held in GITAM University Bangalore campus. She is an effective communicator with excellent interpersonal and logical thinking skills, she has passion towards Data science and Artificial intelligence.

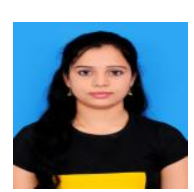

V V Mounika, Velavarthi Venkata Mounika is currently pursuing her bachelor's degree in the stream of computer science and engineering from GITAM deemed to be university, Bengaluru. Her areas of interest include internet of things, bigdata Analytics, Robotic process automation. She is certified in Bigdata Analytics from Dell EMC and is also certified in RPA from Automation Anywhere. She actively participated in various workshops on IoT conducted by GITAM and also has successfully completed a project on Liquid Level Monitoring Using IoT. She has attended Cyber security workshop to identify various malwares conducted by K7 security systems at GITAM University. She is also an active member of the campus robotic club of GITAM University, Bengaluru campus.

Blue Eyes Intelligence Engineering

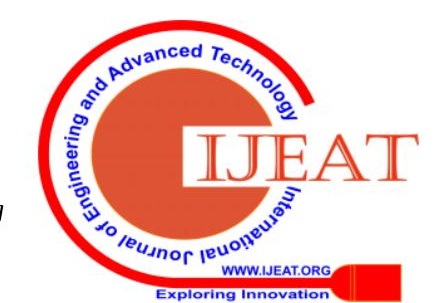

